\title{
Fair and Efficient Scheduling of Variable Rate Links via a Spectrum Server
}

\author{
Roy D. Yates, Chandrasekharan Raman and Narayan B. Mandayam \\ WINLAB, Dept. of Electrical and Computer Engineering \\ Rutgers University \\ Piscataway, NJ \\ \{ryates, chandru, narayan\}@winlab.rutgers.edu
}

\begin{abstract}
We consider a centralized Spectrum Server that coordinates the transmissions of a group of links sharing a common spectrum. Links employ on-off modulation with fixed transmit power when active. In the on state, a link obtains a data rate determined by the signal-to-interference ratio on the link. With knowledge of the link gains in the network, the spectrum server schedules the on/off periods of the links so as to satisfy constraints on link fairness and efficiency. We express fairness constraints as lower bounds on the average minimum rate for each link. Efficiency constraints are expressed as lower bounds on the ratio of the average rate to the average transmit power for each link. Subject to fairness and efficiency constraints, the spectrum server finds a schedule that maximizes the average sum rate. Using a graph theoretic model for the network and a linear programming formulation, the resulting schedules are a collection of time shared transmission modes (sets of active links). In the special case when there is no minimum rate constraint, varying the efficiency constraint can cause the optimal policy to vary from a fixed dominant mode with highest sum rate being operated all the time to time sharing among singleton modes in which just one link is active. We also address the case of maximum common rate scheduling under efficiency constraints. Simulation results are presented to substantiate our findings.
\end{abstract}

\section{INTRODUCTION}

Spectrum regulation has traditionally been driven by advances in technology, from improved filters to the sophisticated logic and radio techniques that created the cellular revolution. More recently, however, a new paradigm has emerged in which regulation has driven technology. The regulatory experiment in open access to spectrum has led to an impressive variety of important technologies and innovative uses. Emerging technologies called "cognitive radio" offer significant potential in terms of better system capacity and service quality, for these systems to adapt to a wide variety of unpredictable conditions.

Cognitive radios, in their simplest form, can recognize the available systems and adjust their frequencies, waveforms and protocols to access those systems efficiently. As a natural consequence, most current research activities are focused on the "design" issues in building such systems [1]. These activities, however, fail to fully reveal the ultimate limits of cognitive behaviour. This is perhaps analogous to a traveller with fluency in a variety of languages. Such fluency is great advantage, but how much greater an advantage is conferred when the traveller understands local conditions and customs, can choose the best language when several are possible, and can find local advisors and information when necessary. Following this analogy, our cognitive radio must do more than communicate with the "local population" on an ad hoc basis to realize its full potential - it must develop a full awareness of a local environment that may span multiple spectrum bands and systems. This implies new discovery processes that are thorough and efficient, and even new classes of "information servers" that provide assistance in the process.

When there exist methods by which cognitive radios can independently discover local information, a variety of physical layer, system and network layer protocols can be applied to allow cooperation and coexistence. However, such levels of cooperation and interoperability may not be possible when multiple services and systems must coexist. In this case, efficient open access to spectrum can be aided by impartial "spectrum servers" [2], [3] which can obtain information about the interference environment through measurements contributed by different terminals, and then offer suggestions for efficient coordination to interested service subscribers. As pointed out in [2], likely neighborhood information could include various levels of time and frequency utilization, descriptions of nodes in a neighborhood, and potentially, spatial positions as well. In fact, the role of such a spectrum server for wireless network coordination is reminiscent of the role of the DHCP (dynamic host configuration protocol) server in the coordination problem that arises among nodes in the Internet.

There are many different ways in which the spectrum server can coordinate a set of radios in a wireless network. Recent work in [4] has considered the role of the spectrum server in scheduling variable rate links while the work in [5] has considered the spectrum server's role in demand responsive pricing and competitive spectrum allocation. Specifically, in [4] the problem of scheduling transmissions for a group of links was considered under the objective of maximizing the sum rate obtained by the links. Issues of fairness were also addressed by deriving scheduling algorithms that resulted in max-min fair [6] and proportional fair [7], [8] rate allocations. In this paper, we introduce the notion of energy efficiency into the scheduling problem and also address the issue of fairness in this context.

Scheduling transmissions in a wireless network has been studied in various contexts. In [9], a joint scheduling and 


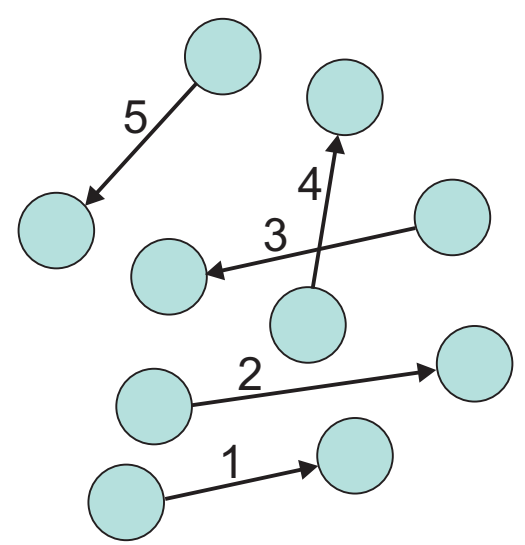

Fig. 1. Graph of network showing the nodes and directed links

power control strategy is proposed to maximize network throughput and energy efficiency of the system. Another variant of this problem is addressed in [10], [11], where the authors look at the cross-layer issues of routing, scheduling and power control. The authors in [12] develop a framework for integrated link scheduling and power control policies to maximize the average network throughput, when each link is subject to an average power constraint and each node is subject to a peak power constraint. The authors assume a model in which the data rate of a link is a linear function of the signalto-interference ratio at the receiver.

In contrast, following our prior work in [4], we consider transmitters with a fixed power on-off modulation and devise schedules that maximize average throughput objectives under energy efficiency constraints. We assume that we obtain a nonzero rate in the links for any non-zero signal-to-interference ratio (SIR). The optimization problem, subject to both energy efficiency and minimum rate constraints in the individual links, is posed as a linear program. If the link gains are known to the spectrum server, it can schedule the transmissions among the links to maximize the system throughput.

The paper is organized as follows. In section II, we describe the system model. The problem formulation and analytical results are described in section III. The schedule which maximizes the common minimum rate is discussed in section IV. The simulation results are presented in section V. We conclude in section VI with some discussions.

Before we explain the system model, we comment on the notation of this paper. We use boldface lowercase characters for vectors and boldface uppercase for matrices. If $\mathbf{a}$ is $\mathbf{a}$ vector, $\mathbf{a}^{T}$ denotes its transpose and $\mathbf{a}^{T} \mathbf{b}=\sum_{i} a_{i} b_{i}$ represents the inner product of the vectors $\mathbf{a}$ and $\mathbf{b}$. The vector of all zeros and all ones are represented by $\mathbf{0}$ and $\mathbf{1}$ respectively.

\section{System Model}

Consider a wireless network with $N$ nodes forming $L$ logical links sharing a common spectrum. The network can be represented as a directed graph $\mathcal{G}(\mathcal{V}, \mathcal{E})$, where the nodes in the network are represented by the set of vertices $\mathcal{V}$ of the graph and the links are represented by a set of directed

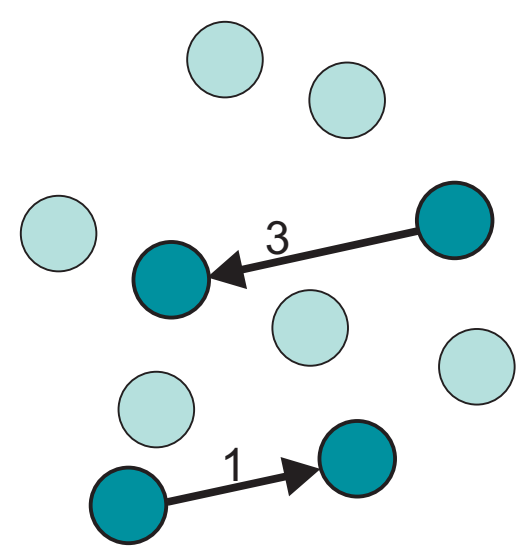

Fig. 2. Graph of network showing transmission mode corresponding to $\left(\begin{array}{llll}1 & 0 & 1 & 0\end{array}\right)$

edges $\mathcal{E}$. Therefore the cardinalities $|\mathcal{V}|=N$ and $|\mathcal{E}|=L$. A directed edge from a node $m$ to node $n$ implies that $n$ wishes to communicate data to node $m$. We consider the scenario where the spectrum server coordinates the activity of the set of $L$ links to share the spectrum efficiently.

Define the set $\mathcal{T}=\{0,1, \ldots, M-1\}$ of transmission modes, where $M=2^{L}$ denotes the number of possible transmission modes. Then the mode activity vector $\mathbf{t}_{i}$ of mode $i$ is a binary vector, indicating the on-off activity of the links. If $\mathbf{t}_{i}=\left(t_{1 i}, t_{2 i}, \ldots, t_{L i}\right)$ is a mode activity vector, then

$$
t_{l i}= \begin{cases}1, & \text { link } l \text { is active under transmission mode } i, \\ 0, & \text { otherwise }\end{cases}
$$

Note that there are $M$ possible transmission modes including the mode in which all links are off. Figure 1 shows a representative network and Figure 2 shows particular transmission mode for the set of links.

Let the transmitter power on a link $l$ be $P_{l}$. If $G_{l k}$ is the link gain from the transmitter of link $k$ to the receiver of link $l$ and $\sigma_{l}^{2}$ is the noise power at the receiver of link $l$, the SIR $\gamma_{l i}$ at the receiver of link $l$ in transmission mode $i$ is given by

$$
\gamma_{l i}=\frac{t_{l i} G_{l l} P_{l}}{\sum_{k \in \mathcal{E}, k \neq l} t_{k i} G_{l k} P_{k}+\sigma_{l}^{2}} .
$$

The link gain between a transmitter and receiver takes into account the path loss and attenuation due to shadow fading. We assume that the link gains between each transmitter and receiver are known to the spectrum server. The data rate in each link depends on the SIR in that link. We assume that the transmitter can vary its data rate, possibly through a combination of adaptive modulation and coding. In particular, for a given mode, the transmitter and receiver on a link employ the highest rate that permits reliable communication given the link SIR in that mode. For purposes of this study, we assume that the transmission of other links are treated as Gaussian noise and that a transmission on link $l$ is reliable in a given mode $i$ with a data rate

$$
c_{l i}=\log \left(1+\gamma_{l i}\right) .
$$


We emphasize here that we do not consider any minimum SIR threshold required at each receiver, i.e., associated with each transmission mode $i$, a non-zero $\gamma_{l i}$ defines some rate on the link $l$. Let $x_{i}$ be the fraction of time that transmission mode

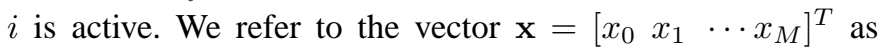
a schedule without precise specification of sequence of active modes. The average data rate in link $l$ is the time average of the data rates of all the transmission modes that include link $l$ and is given by

$$
r_{l}=\sum_{i} c_{l i} x_{i}
$$

Similarly, the average power $\bar{P}_{l}$ used by link $l$ is obtained as the time average of the power used by link $l$ in all the transmission modes. Hence,

$$
\bar{P}_{l}=\sum_{i} P_{l} t_{l i} x_{i}
$$

We define the energy efficiency $\epsilon_{l}$ of a link as the ratio of the average rate obtained by link $l$ to the average power $\bar{P}_{l}$ expended by the link. Hence,

$$
\epsilon_{l}=\frac{r_{l}}{\bar{P}_{l}}
$$

We can view the efficiency of a link as the data rate obtained per unit of power used in transmission on the link. Note that the efficiency depends on the schedule $\mathbf{x}$ and that $\epsilon_{l}$ is undefined if link $l$ does not transmit during that schedule.

The highest efficiency of a link is attained when it transmits in isolation. The modes corresponding to a link $l$ transmitting in isolation without interference from other links is referred to as the link $l$ singleton mode. If the system were to use only the link $l$ singleton mode, link $l$ obtains SINR (in fact SNR)

$$
\bar{\gamma}_{l}=\frac{G_{l l} P_{l}}{\sigma_{l}^{2}}
$$

and efficiency

$$
\bar{\epsilon}_{l}=\frac{\log \left(1+\bar{\gamma}_{l}\right)}{P_{l}} .
$$

For any schedule in which link $l$ is active, its efficiency $\epsilon_{l}$ must satisfy $\epsilon_{l} \leq \bar{\epsilon}_{l}$ since among the all the transmission modes, the link obtains its maximum rate in the singleton mode as there are no other interfering users that bring down the rate.

In this paper, we are interested in obtaining efficient scheduling strategies which maximize the sum rate in the links by ensuring that all links operate above a certain threshold efficiency $\epsilon_{0}$, i.e., $\epsilon_{l} \geq \epsilon_{0}$ for all links $l$. Hence from (6),

$$
\sum_{i} c_{l i} x_{i} \geq \epsilon_{0} \sum_{i} P_{l} t_{l i} x_{i}
$$

We denote $\mathbf{P}=\operatorname{diag}\left(P_{1}, P_{2}, \ldots, P_{L}\right)$ to be the $L \times L$ diagonal matrix of transmit powers of the individual links. We define the $L \times M$ matrix $\mathbf{C}=\left[\begin{array}{lll}\mathbf{c}_{1} & \mathbf{c}_{2} \ldots \mathbf{c}_{M}\end{array}\right]$ such that its $j$ th column $\mathbf{c}_{j}=\left[c_{1 j}, c_{2 j}, \ldots, c_{L j}\right]^{T}$ contains the rate obtained by each link in mode $j$. Let $\mathbf{T}=\left[\begin{array}{lll}\mathbf{t}_{1} & \mathbf{t}_{2} \ldots \mathbf{t}_{M}\end{array}\right]$ denote the $L \times M$ binary matrix, which contains the transmission mode vector $\mathbf{t}_{j}$ as column $j$. Thus, equation (9) can be written in vector form as

$$
\mathbf{C x} \geq \epsilon_{0} \mathbf{P T x} .
$$

In the following section, we present the linear program (LP) for fair and efficient sum rate maximization.

\section{FAIR AND EFFICIENT MAXIMUM SUm RATE SCHEDULING}

We are interested in an efficient schedule that maximizes the sum of the average data rates over all links $l=1,2, \ldots, L$, subject to constraints on the minimum rate for each link. The optimization problem can be posed as the LP:

$$
\begin{aligned}
\max & \mathbf{1}^{T} \mathbf{r} \\
\text { subject to } & \mathbf{r}=\mathbf{C} \mathbf{x}, \\
& \mathbf{r} \geq r_{\min } \mathbf{1}, \\
& \mathbf{r} \geq \epsilon_{0} \mathbf{P} \mathbf{T} \mathbf{x}, \\
& \mathbf{1}^{T} \mathbf{x} \leq 1, \\
& \mathbf{x} \geq \mathbf{0} .
\end{aligned}
$$

The objective function $\mathbf{1}^{T} \mathbf{r}=\sum_{i} r_{i}$ is the sum of average rates of the individual links. The constraint (11b) represents the minimum rate constraint and (11c) is the efficiency constraint.

The variables in the LP (11) are $\mathbf{r}$ and $\mathbf{x}$. Rewriting the LP in terms of the variable $\mathbf{x}$ only, we get

$$
\begin{aligned}
c_{\text {opt }}\left(r_{\min }, \epsilon_{0}\right)=\max & \mathbf{1}^{T} \mathbf{C x} \\
\text { subject to } & \mathbf{C x} \geq r_{\min } \mathbf{1} \\
& \left(\mathbf{C}-\epsilon_{0} \mathbf{P T}\right) \mathbf{x} \geq \mathbf{0} \\
& \mathbf{1}^{T} \mathbf{x} \leq 1 \\
& \mathbf{x} \geq \mathbf{0} .
\end{aligned}
$$

We now consider the special case $r_{\min }=0$ when there is no minimum rate requirement for any of the links. Before proceeding, we observe that $c_{l i}=0$ if and only if $t_{l i}=0$. Thus for sufficiently small efficiency threshold $\epsilon_{0}, \mathbf{C}-\epsilon_{0} \mathbf{P T}$ is a nonnegative matrix. In this case, the constraint (12b) is inactive in that it is trivially satisfied for all nonnegative $\mathbf{x}$.

In the absence of the efficiency constraint, it was shown in [4] that the optimal schedule for the problem (12) with $r_{\min }=0$ is to operate only a transmission mode $d$ with maximum sum rate. The optimal objective value is the maximum column sum of the rate matrix $\mathbf{C}$. We refer to mode $d$ as the dominant mode and we denote by $\hat{\mathbf{x}}=\left[\begin{array}{llllll}0 & \cdots & 0 & 1 & 0 & \cdots\end{array}\right]^{T}$ the schedule that supports exclusive use of mode $d$. In addition, we use $\mathcal{D}$ to denote the set of active links in the dominant mode. For links $l \in \mathcal{D}$, we use $\hat{\epsilon}_{l}$ to denote the efficiency of the link under the schedule $\hat{\mathbf{x}}$.

When the efficiency threshold $\epsilon_{0}$ satisfies

$$
\epsilon_{0} \leq \hat{\epsilon}=\min _{l \in \mathcal{D}} \hat{\epsilon}_{l}
$$

all links in the dominant mode will meet the efficiency constraint under schedule $\hat{\mathbf{x}}$. In this case, schedule $\hat{\mathbf{x}}$, corresponding to exclusive use of the dominant mode, remains optimal. When the efficiency threshold $\epsilon_{0}$ passes $\hat{\epsilon}$, the optimal 
schedule may continue to employ the dominant mode, but other modes also must be scheduled to boost the efficiency of the least efficient links in the dominant mode. Eventually, the problem becomes infeasible when $\epsilon_{0}$ exceeds $\bar{\epsilon}=\max _{l} \bar{\epsilon}_{l}$, the maximum efficiency in a singleton mode.

Depending on the geometry of the links, the dominant transmission mode can be a single active link or a collection of geographically separated links. However, the geographic separation of links that is typically associated with the dominant mode is consistent with those links in the dominant mode having high efficiency. The consequence is that enforcing an efficiency constraint typically has little impact until the efficiency constraint is very stringent. This implies that the links that are not a part of the dominant transmission mode are starved. So, the system is not fair in terms of providing non-zero data rates to all the links.

In order to offset the inherent unfairness in the above solution, we introduce a non-zero minimum rate constraint in the problem (12). When we impose a non-zero minimum rate $r_{\min }$ on all links, the optimal schedule may change. Once again, for small values of the threshold $\epsilon_{0}$, the efficiency constraint is inactive. In such cases, the optimal schedule balances the use of the dominant mode against modes that provide non-zero rates to otherwise disadvantaged links. This case is examined in greater detail in [4]. Increasing the threshold $\epsilon_{0}$ gradually eliminates modes in which links are active in the presence of significant interference. For high values of $\epsilon_{0}$, the system tends to use the high-efficiency singleton modes, although this can have a significant penalty in terms of the sum rate.

\section{MAXIMUM COMMON RATE SCHEDULING}

The maximum sum rate scheduling is biased towards links that have the best quality (i.e., least interference) and is unfair to the other links that are not a part of the dominant transmission mode. To address this, we present the maximum common rate schedule in this section and we conjecture that this schedule may result in the max-min fair rate allocation.

Definition 1: A vector of rates $\mathbf{r}$ is said to be max-min fair if it is feasible and for each $l \in \mathcal{E}, r_{l}$ cannot be increased while maintaining feasibility without decreasing $r_{l^{\prime}}$ for some link $l^{\prime}$ for which $r_{l^{\prime}} \leq r_{l}$. Formally, for any other feasible allocation $\tilde{\mathbf{r}}$, with $\tilde{r}_{l}>r_{l}$, there must exist some $l^{\prime}$ such that $\tilde{r}_{l^{\prime}}<r_{l^{\prime}} \leq r_{l}$.

In the context of flow control of sources in a communication network, iterative algorithms for computing max-min fair rate vectors exist [6]. Such iterative algorithms use a 'progressive filling' technique that starts with all rates equal to zero and increases the rates until one or several link capacity limits are reached. In order to obtain an energy efficient max-min fair schedule, we begin by formulating the LP to maximize the minimum common rate in all the links subject to energy

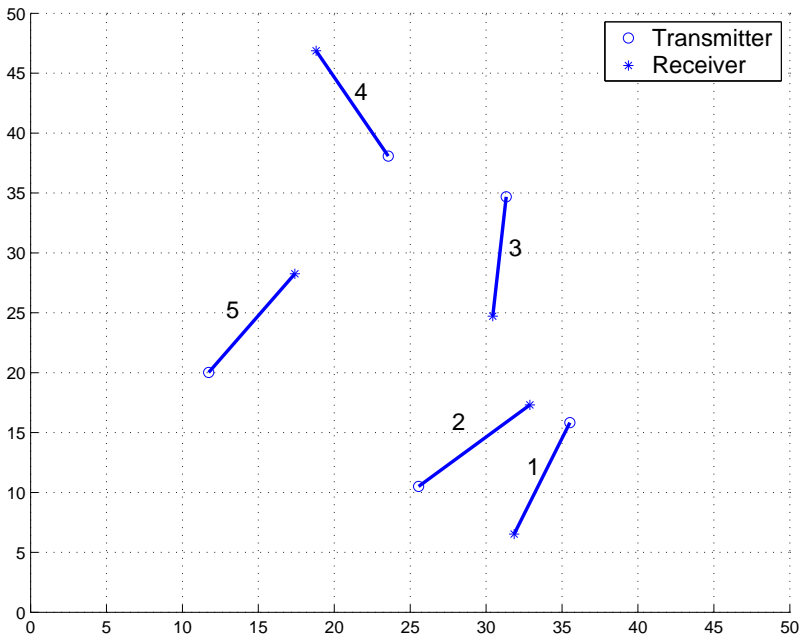

Fig. 3. Set of five links each of length $d=10$.

efficiency constraints.

$$
\begin{array}{ll}
r^{*}=\max & r_{\min } \\
\text { subject to } & \mathbf{r}=\mathbf{C} \mathbf{x}, \\
& \mathbf{r} \geq r_{\min } \mathbf{1}, \\
& \mathbf{r} \geq \epsilon_{0} \mathbf{P} \mathbf{T} \mathbf{x}, \\
& \mathbf{1}^{T} \mathbf{x}=1, \\
& \mathbf{x} \geq \mathbf{0} .
\end{array}
$$

We conjecture that if the link gains $G_{l j}$ are all non-zero, then the LP (14) which maximizes the minimum common rate among the links subject to efficiency constraint, results in all links obtaining the same rate $r^{*}$, i.e., $\mathbf{r}^{*}=r^{*} \mathbf{1}$. In the absence of an efficiency constraint, such a claim was proven in [4]. With an efficiency constraint, the proof in [4] does not follow directly. However, our simulation results suggest that the claim is likely to hold. If this were the case, then the rate allocation $r^{*} 1$ will be the max-min fair rate allocation.

\section{Simulation RESUlts}

We present some simulation results in this section. Though the analytical results are true for more general cases, we present simulation results for some specific cases to illustrate our findings. The simulation set-up is a $50 \times 50$ grid as shown in Figure 3. The links are of fixed lengths and placed at random locations in the grid. The interference gain $G_{l j}$ between the transmitter of link $j$ and the receiver of a link $l$ is given by $G_{l j}=d_{l j}^{-4}$, where $d_{l j}$ is the separation distance between the transmitter and receiver. The transmit powers are fixed for all transmissions and the link geometries are characterized through the signal-to-noise ratio (SNR) at the receiver for that link (in the absence of interference).

In the case of maximum sum rate scheduling with no minimum rate constraint, for a fixed $\epsilon_{0}=\epsilon$, the transmission mode with the highest sum rate is chosen. The links which are not a part of the dominant transmission mode are not operated 


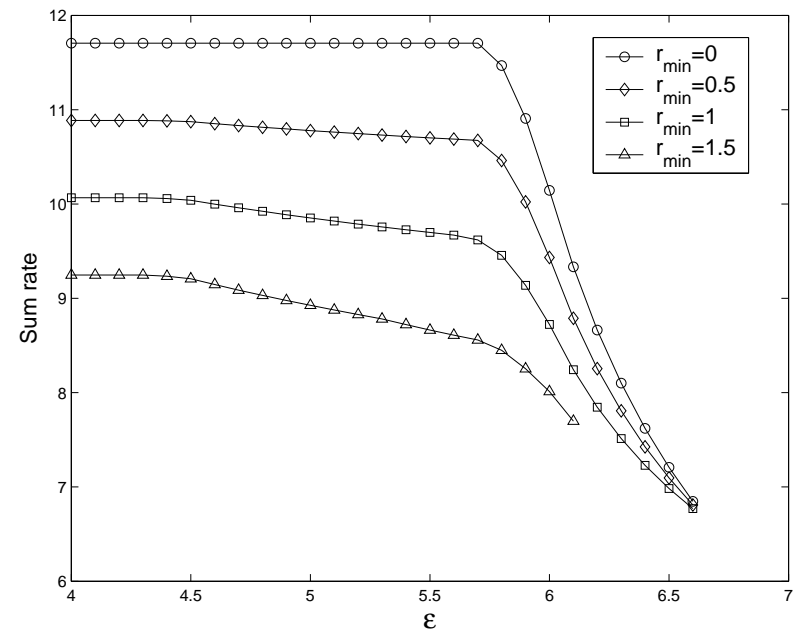

Fig. 4. Variation of sum rate with efficiency $\epsilon$

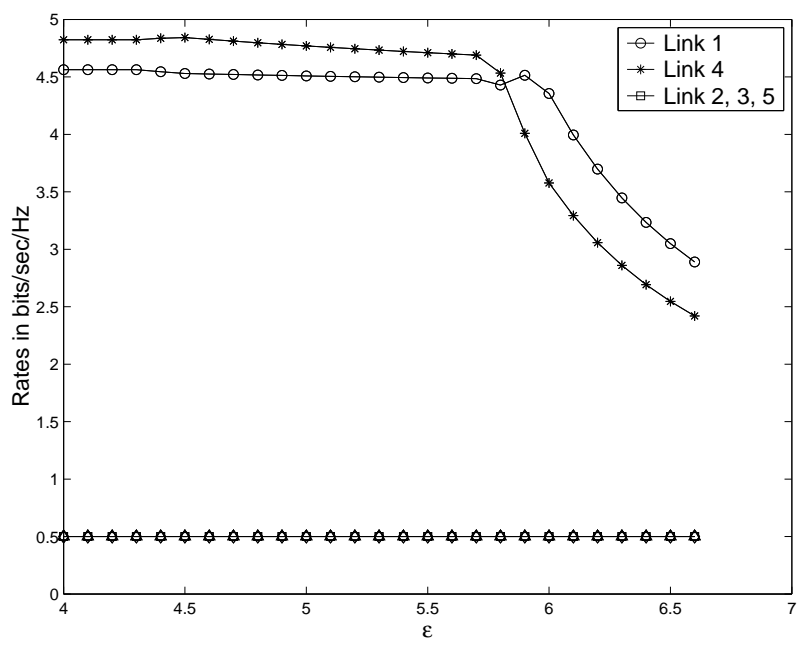

Fig. 5. Variation of rates of individual links with efficiency $\epsilon$ for $\mathbf{r}_{\min }=0.5$

at all. As we increase $\epsilon$, the sum rate remains constant until a certain threshold value, say $\epsilon_{t h}$. The value of $\epsilon_{t h}$ is the efficiency of the weakest (in terms of link quality) link in the dominant mode. For $\epsilon>\epsilon_{t h}$, single link modes corresponding to some of the links in the dominant mode are operated in order to satisfy the efficiency constraints on those links. The variation of sum-rate with $\epsilon$ is shown in Figure 4.

In the case of maximum sum rate scheduling with nonzero minimum rate constraint, we see that more than one transmission mode is operated since there is a minimum rate requirement for each link. As with the case with zero minimum rate constraint, for a given degree of fairness (i.e., a specified $\left.r_{\min }\right)$, there is relatively little penalty for requiring efficiency, until the breakpoint where the required efficiency approaches the efficiency of the link in isolation. For larger values of $r_{\min }$, the efficiency of the single link modes decreases. This is illustrated in Figure 4. Notice that for non-zero $r_{\min }$ values, there is some loss in sum rate because of the fairness introduced

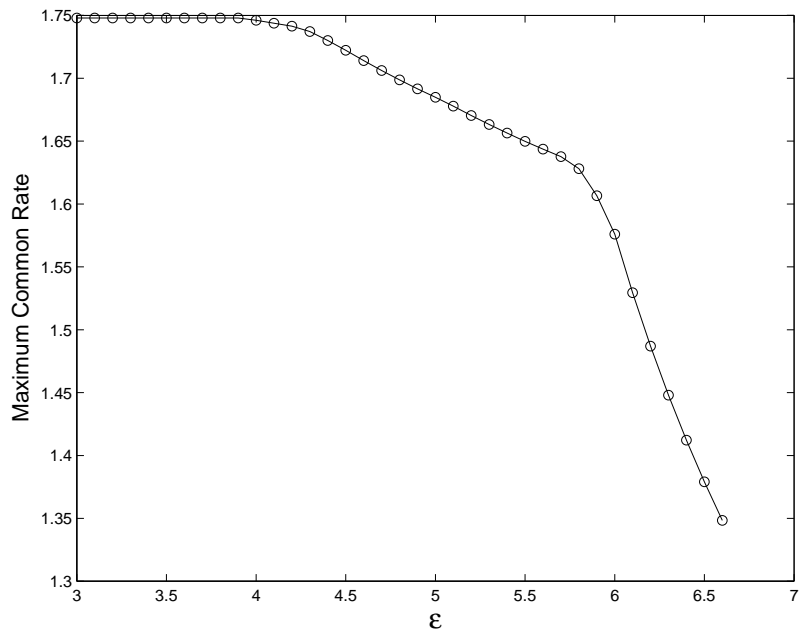

Fig. 6. Variation of the maximum common rate $r^{*}$ with efficiency $\epsilon$

by $r_{\min }$. However, similar to the case when $r_{\min }=0$, when $\epsilon_{0}$ crosses a threshold, the penalty is sum rate increases as efficiency increases.

Figure 5 shows the variation of rates of individual links with the efficiency. For lower values of $\epsilon$, the dominant mode $\{1,4\}$ is operated to maximize the sum rate and the links which are not a part of the dominant mode are scheduled for just enough time to satisfy their minimum rate constraint. When higher efficiency is required, the rates of links in the dominant mode reduces until the singleton modes are not efficient any more.

Figure 6 shows the trade-off between fairness (determined by the maximum common rate among the links) and efficiency. As the link efficiency $\epsilon$ increases, we see that the maximum common rate among the links decreases.

\section{DisCUSSION AND CONCLUSION}

We observe that from low to moderate values of the efficiency threshold $\epsilon_{0}$, the efficiency constraint made little difference because the high throughput solutions were also the high efficiency solutions. However, for high values of $\epsilon_{0}$, the penalty paid in terms of throughput is large. But in a schedule with only fairness constraints, we observed in [4] that the loss in sum rate is gradual with increase in the minimum required rate $r_{\min }$.

The scheduling algorithm discussed in this paper, is typically run on the centralized server. In order to simplify the algorithm, the spectrum server can dispense with the efficiency constraints in scheduling links. Since efficiency and high throughput appear to be positively correlated, distributed scheduling algorithms may be able to use the efficiency constraints to steer links to efficient schedules. In this case, a spectrum server can announce an efficiency target and links will be pushed towards schedules that employ efficient modes. Such distributed algorithms may involve links reporting the interference seen by them from all other links and their own efficiency. 
To summarize, we considered a centralized Spectrum Server that coordinates the transmissions of a group of links in a wireless network, which is modelled as a directed graph. The problem of maximizing the sum rate in all the links subject to minimum rate constraints and energy efficiency constraints was posed as a linear program. With knowledge of the link gains in the network, the spectrum server scheduled the on/off periods of the links so as to satisfy constraints on link fairness and efficiency. Subject to fairness and efficiency constraints, we derived two classes of schedules; one that maximized the average sum rate and the other that maximized the common rate. In the special case when there was no minimum rate constraint, varying the efficiency constraint caused the optimal policy to vary from a fixed dominant mode with highest sum rate being operated all the time to time sharing among singleton modes in which just one link is active.

\section{ACKNOWLEDGEMENT}

This work is supported in part by the NSF under grant number NeTS-0434854 and by the Defense Spectrum Office (DSO) of the Defense Information Systems Agency.

\section{REFERENCES}

[1] A. Sahai, N. Hoven, and R. Tandra, "Some fundamental limits on cognitive radio," in Proc. of Allerton Conf. on Comm., Control and Computing, Oct 2004

[2] N. Mandayam, "Cognitive algorithms and architectures for open access to spectrum," Conf. on the Economics, Technology and Policy of Unlicensed Spectrum, East Lansing, MI, May 2005. http://quello.msu.edu/conferences/spectrum/program.htm.

[3] M. Buddhikot, P. Kolodzy, S. Miller, K. Ryan, and J. Evans, "DIMSUMNet: New directions in wireless networking using coordinated dynamic spectrum access," in IEEE WoWMoM05, June 2005.

[4] C. Raman, R. Yates, and N. Mandayam, "Scheduling variable rate links via a spectrum server," in Proc. IEEE DySPAN 2005, 2005. Baltimore, MD.

[5] O. Ileri, D. Samardzija, T. Sizer, and N. Mandayam, "Demand responsive pricing and competitive spectrum allocation via a spectrum policy server," in Proc. IEEE DySPAN 2005, Nov 2005. Baltimore, MD.

[6] D. Bertsekas and R. Gallager, Data Networks. Prentice-Hall, 1992.

[7] P. Viswanath, D. Tse, and R. Laroia, "Opportunistic beamforming using dumb antennas," IEEE Trans. Info. Theory, vol. 48, pp. 1277-1294, Jun 2002.

[8] S. Shakkottai and A. Stolyar, "Scheduling for multiple flows sharing a time-varying channel: The exponential rule," American Mathematical Society Translations, vol. 207, 2002.

[9] T. Elbatt and A. Ephremides, "Joint scheduling and power control for wireless ad-hoc networks," IEEE Trans. Wireless Commun., vol. 3, pp. 74-85, Jan 2004.

[10] R. L. Cruz and A. V. Santhanam, "Optimal routing, link scheduling and power control in multi-hop wireless networks," IEEE Infocom, 2003.

[11] R. Bhatia and M. Kodialam, "On power efficient communication over multi-hop wireless networks: Joint routing, scheduling and power control," in IEEE Infocom, 2004

[12] R. L. Cruz and A. V. Santhanam, "Optimal link scheduling and power control in CDMA multihop wireless networks," in IEEE Globecom, pp. 52-56, 2002. 